\title{
Keyword Index Volume 16 (2012)
}

\begin{tabular}{|c|c|c|c|}
\hline applications & 683 & \multicolumn{2}{|c|}{$451,513,581,717$} \\
\hline association rule mining & 49 & data stream mining & 803 \\
\hline \multirow[t]{2}{*}{ atmospheric pollution } & 153 & decision combining and fusion & 879 \\
\hline & & decision lists & 247 \\
\hline bag of spatio-temporal words & 537 & decision rules & 665 \\
\hline Bayesian networks & 199 & decision trees & 247,649 \\
\hline Bayesian optimization algorithm & 199 & descriptive multivariate analysis & 279 \\
\hline Bayesian score & 847 & deviation based outlier mining method & 487 \\
\hline biometrics & 409 & deviation detection & 487 \\
\hline bipartite graphs & 93 & differential evolution & 3 \\
\hline \multirow[t]{2}{*}{ Boolean reasoning } & 915 & dimensionality reduction & 69 \\
\hline & & discretization & 915 \\
\hline case-based reasoning & 199 & distribution rules & 25 \\
\hline change mining & 93 & divide-and-conquer technique & 953 \\
\hline Chi-square test & 847 & drug discovery & 137 \\
\hline class association rules & 49 & & \\
\hline \multicolumn{2}{|c|}{$247,305,351,551,633,665$} & ensemble classifier & 233 \\
\hline classification ensemble & 763 & ensemble classifiers & 777 \\
\hline closed frequent subtrees & 953 & ensemble design heuristic & 233 \\
\hline closed set & 581 & error tolerance dissimilarity function & 487 \\
\hline clustering & 37,703 & evolving databases & 365 \\
\hline clustering evolution & 93 & experimental test bench & 279 \\
\hline clustering multiple data streams & 69 & & \\
\hline co-EM & 745 & feature extraction & 409 \\
\hline \multicolumn{2}{|l|}{ complementary distribution binary particle } & feature ranking & 879 \\
\hline swarm optimization & 183 & feature selection & $167,183,265,305,717$ \\
\hline comprehensibility & 247 & feature space discriminating & 537 \\
\hline concept drift & 803 & feature weighting & 537 \\
\hline conceptual graph interchange format & 487 & features selection & 879 \\
\hline concise representation & 581 & Formal Concept Analysis & 581 \\
\hline context-awareness & 803 & Forward stepwise regression & 265 \\
\hline continuous optimization & 3 & fraud detection & 467 \\
\hline co-training & 745 & frequent items & 513 \\
\hline \multirow[t]{2}{*}{ customer relationship management } & 265 & frequent itemset & 581 \\
\hline & & frequent sequence & 451 \\
\hline data a & 409 & functional dependencies & 365 \\
\hline data mining & 5,365 & fuzzy systems & 633 \\
\hline
\end{tabular}




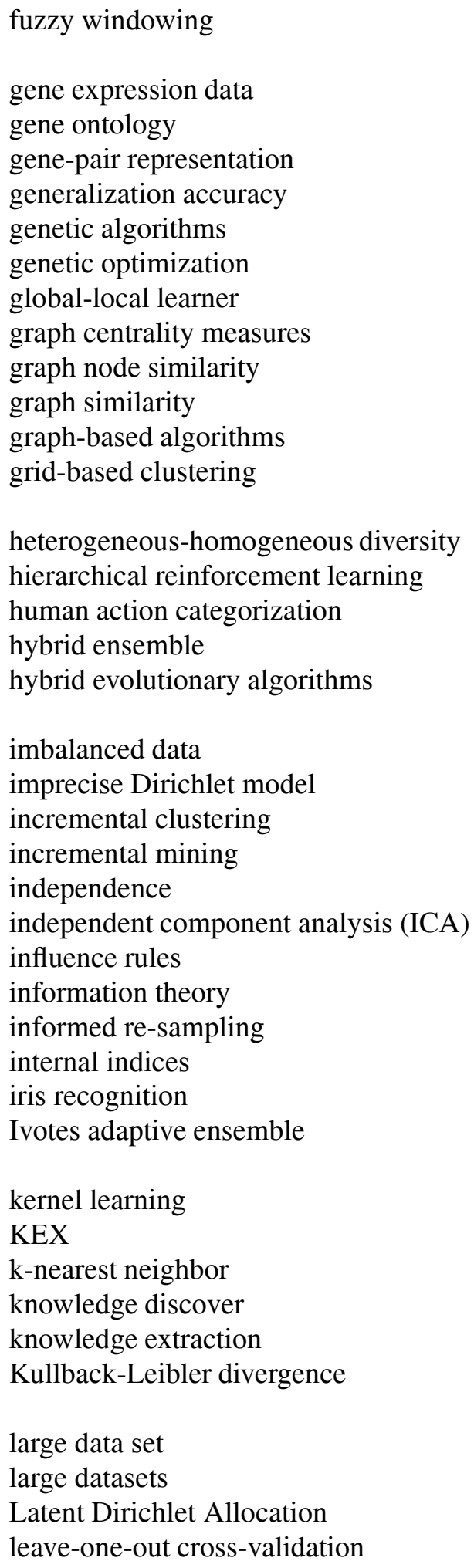

279 leverage 25

linear transform 383

827 LISp-Miner 665

827 local spatio-temporal features 537

827 long-term 427

633

167 machine learning 3,233, 305, 427, 827

763 machine translation 633

233 maintenance 365

113 memetic algorithms 3

865 meta-learning 763

865 microarray analysis 717

211 minimal generator 581

69 mixture models 467

monitoring 93

233 multidimensional scaling 153

113 multimodal analysis 683

537 multimodal annotation 683

233 multiple correspondence analysis 279

3 Multiple logistic regression 265

multi-view semi-supervised learning

$847 \quad$ Neural networks 265

69 node connection graph stability 113

953 non-uniformly sampled data 993

847 numerical association rules 25

$\begin{array}{ll}409 & \\ 513 & \text { ontology }\end{array}$

879 optimal association rules 25

777 optimisation 305

703 oracle coaching 247

409 otimization 199

777 overlapping clustering 211

351 particle swarm optimisation 305

665 particle swarm optimization 167,915

183 pattern analysis 993

365 pattern growth 451

513 periodicity detection 993

467 predictive models 763

principal component analysis $\quad 279$

897 prior knowledge injection 113

649 privacy preserving data mining 933

467 protein-drugs network 137

183 protein-protein interactions 137 
random selection

ranking

recurring concepts

regression

relative Newton method

robust Bayesian mixtures

robust clustering

robust Dirichlet process mixtures

rough sets theory

rule-weight

sampling

sanitization

security systems

semantic similarity

semi-supervised ensemble

sequence mining

sequential search algorithms

similarity measure

skill acquisition

social networks

SPIDER method

state-transition diagrams

statistical tests

stock market data

stopping rules

Stream join

suffix tree

supervised classification

support vector learning

Support Vector Machines

SVM
897 telecommunications 467

383 text categorization $\quad 879$

803 text classification 879

427 text mining 487

409 text outliers 487

969 time series 993

969 train door system 279

969 transitions 93

915 travel time prediction 427

633 tree mining 953

tumor classification $\quad 717$

745

933 ubiquitous knowledge discovery 803

409 unfolding 153

827 user profiling 467

745 utility and frequent itemset 933

451 utility mining 933

167

865 variable selection 551

113 variational inference 969

137 visual analytics 683

777 visualization 683

683 visuatizion

847 web content mining 327

993 web site keywords 327

703 web site text content 327

69 web usage mining $\quad 327$

993 weighted scoring rule 383

49, 649 weighting method 383

351 word sense disambiguation 633 